\title{
Kinetic roughening in growth models with diffusion in higher dimensions
}

\author{
P. Šmilauer ${ }^{a \text {, 出 }}$ and M. Kotrla ${ }^{b}$ \\ ${ }^{a}$ Interdisciplinary Research Centre for Semiconductor Materials, Imperial College, London \\ SW7 2BZ, United Kingdom \\ ${ }^{b}$ Institute of Physics, Academy of Sciences, Na Slovance 2, \\ 18040 Praha 8, Czech Republic \\ PACS 05.70L, 05.40, 68.55
}

\begin{abstract}
We present results of numerical simulations of kinetic roughening for a growth model with surface diffusion (the Wolf-Villain model) in $3+1$ and $4+1$ dimensions using lattices of a linear size up to $L=64$ in $3+1 \mathrm{D}$ and $L=32$ in $4+1 \mathrm{D}$. The effective exponents calculated both from the surface width and from the height-height correlation function are much larger than those expected based on results in lower dimensions, due to a growth instability which leads to the evolution of large mounded structures on the surface. An increase of the range for incorporation of a freshly deposited particle leads to a decrease of the roughness but does not suppress the instability.
\end{abstract}

Kinetic roughening in growth models has been extensively studied in recent years [1]. Two approaches are mainly used: a macroscopic, continuum theory based on stochastic differential equations, and numerical simulations of discrete models. The width (roughness) of a growing surface, $w(t, L)=\left\langle\sqrt{\overline{h^{2}}-\bar{h}^{2}}\right\rangle$ (the variance of the surface height profile $h(\mathbf{x}, t)$ ), obeys the dynamical scaling law $w(t, L) \propto L^{\zeta} f\left(t / L^{z}\right)$ as a function of time $t$ and the system size $L$, where $f(y) \rightarrow$ const as $y \rightarrow \infty$ and $f(y) \propto y^{\beta}, \beta=\zeta / z$ as $y \rightarrow 0$. The asymptotic behavior of the surface width is characterized by the exponents $\zeta$ and $z$ (or $\zeta$ and $\beta$ ) which depend on the universality class of the model used and on the spatial dimension $d=d^{\prime}+1$, $d^{\prime}$ being the surface dimension.

Recently, a new class of models with diffusion (or, more precisely, post-deposition relax-

\footnotetext{
${ }^{1}$ On leave from Institute of Physics, Academy of Sciences, Cukrovarnická 10, 16200 Praha 6, Czech Republic
} 
ation) has been investigated [2, 3, 4. A physical mechanism behind the surface smoothing in these growth models is surface diffusion controled by the nearest-neighbor bonding energy and it was supposed that these simplified models should mimic the situation in growth by molecular beam epitaxy. The effective scaling exponents obtained from simulations of such models are often compared to the exponents obtained using approximate analytical methods [2, 4, 5] from differential equations of the form

$$
\frac{\partial h(\mathbf{x}, t)}{\partial t}=-\nabla \cdot \mathbf{j}(\mathbf{x}, t)+\eta(\mathbf{x}, t)
$$

where the current $\mathbf{j}(\mathbf{x}, t)$ is a function of the derivatives of $h(\mathbf{x}, t)$. (Note, however, that the connection between microscopic computer models and continuum equations is far from being straightforward.) Wolf and Villain (WV) [3] formulated a discrete growth model in which a particle arriving on the surface relaxes in a local neighborhood to increase the number of bonds to nearest neighbors. If there is no possibility to increase the number of nearest neighbors, the particle stays at the initial position. First numerical simulations of the WV model in 1+1 D [3] yielded exponents in surprising agreement with those of the linear differential equation $\left(\mathbf{j} \propto \nabla \nabla^{2} h\right)$. However, new extensive simulations [6] revealed a crossover to intermediate behavior with the exponents corresponding to a nonlinear equation, $\mathbf{j} \propto \nabla(\nabla h)^{2}$, and indicated a further crossover to Edwards-Wilkinson behavior $(\mathbf{j} \propto-\nabla h)$, the second crossover being in agreement with recent calculations by Krug et al. [7] using the inclination-dependent diffusion current.2 Simulations in $2+1 \mathrm{D}$ [8, 6] gave exponents in agreement with those obtained from the nonlinear equation $(\zeta=(5-d) / 3, \beta=(5-d) /(7+d))$. Note that simulations of a full-diffusion (FD) model, in which all surface atoms can move not only immediately after deposition, but during the whole simulation with the rates determined by nearest-neighbor bonding [9], yielded exponents of the nonlinear equation in $1+1,2+1$, and $3+1$ D.

\footnotetext{
${ }^{2}$ Recent results show that the asymptotic behavior of the WV model is of the Edwards-Wilkinson type. However, the correct description of the intermediate behavior is not known at the moment, in particular since the values of the exponents $\zeta^{\mathrm{c}}$ calculated at this regime from the correlation function (see below) correspond to an equation with a nonlinear term $\nabla(\nabla h)^{3}$ in $1+1 \mathrm{D}$, but to an equation with a nonlinear term $\nabla^{2}(\nabla h)^{2}$ in $2+1 \mathrm{D}$ and cannot be explained simultaneously by any of the models considered so far $[$ ]].

${ }^{3}$ The WV model and the FD model are of course different. The Laplacian term should not be present in the FD model equation where the hopping probability depends only on the initial coordination. However, we have found that in $1+1$ dimensions there is very close correspondence in behavior of both models for quite a long time interval with the radius of incorporation $R_{i}$ (described below) corresponding to the temperature in the FD model.
} 
In order to understand the situation better and to check the universality class of the model, we have performed extensive simulations of the WV model in higher unphysical dimensions. Below, we present our results in $3+1$ and $4+1 \mathrm{D}$. The model under study is a straightforward generalization of the model investigated in the lower dimensions [3, 6 , 8]. A particle is deposited at a randomly selected site on the surface and then relaxes to a position with a highest coordination (the number of nearest neighbors) $v$, according to the following rules. The particle relaxes to a site with the highest coordination $v_{\max }$ of all the sites examined if this is also higher than the coordination $v_{\text {init }}$ of the initial site of incidence, $v_{\max }>v_{\text {init }}$. If there are several sites with $v=v_{\max }>v_{\text {init }}$, a random choice is made. If there is no site with a coordination higher than $v_{\text {init }}$, i.e. $v_{\max }=v_{\text {init }}$, the particle stays at the initial position. The solid-on-solid condition is obeyed in the model, i.e., no overhangs or bulk vacancies are allowed. At first we have restricted the possible sites for the relaxation to nearest neighbors only.

The simulations in higher dimensions are much more demanding on computer time not only because of more degrees of freedom but also due to very slow dynamics of the model (the dynamical exponent predicted for, e.g., the nonlinear model, $z=(7+d) / 3$, is large and increases with the spatial dimension). Therefore, one has to deposit a large number of layers to reach the stationary state characterized by the saturated surface width. The maximal linear system sizes for which we were able to measure the saturated surface roughness were $L=45$ (we can only estimate it for $L=64$ ) in $3+1 \mathrm{D}$ and $L=16$ in $4+1 \mathrm{D}$. Exponent $\beta_{\mathrm{eff}}$ was calculated using data for the largest systems, $L=64(3+1 \mathrm{D})$ and $L=24(4+1 \mathrm{D})$. Due to the enormous computer time required we averaged over several independent runs only.

In contrast to the situation in $1+1 \mathrm{D}$ and $2+1 \mathrm{D}$ [6, 8], we have found in the higher dimensions unexpectedly rapid roughening with the effective exponent $\beta_{\text {eff }}$ showing an initial

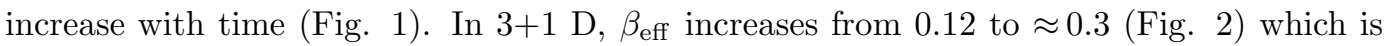
much larger than the value predicted by any of the above-mentioned continuum equations. We have analyzed our data using a linear fit to 7 successive points on the double logarithmic scale (Fig. 2). Maximal values of the effective exponent $\beta_{\mathrm{eff}}(L)$ for different system sizes $L=32-64$ are still slightly increasing which indicates that the size $L=64$ is still not large enough to measure the correct value of the effective exponent. In Fig. 1, the dependence of the saturated surface width on the system size is shown as well. The exponent $\zeta_{\text {eff }}$ increases with the system size from $\zeta_{\text {eff }} \approx 0.47$ for $L=4-8$ to $\zeta_{\text {eff }} \approx 1.25$ for $L=32-45$; this is again 
much larger than the values predicted by any of the continuum equations. It seems that after the initial increase for $L=4-16, \zeta_{\text {eff }}$ remains approximately constant. A linear fit for $L=16-64$ yields $\zeta_{\text {eff }} \approx 1.2$. The corresponding value of the dynamical exponent $z$ is about 4. One can also estimate $z$ from the system-size dependence of the time after which the surface width saturates. In this way we obtain $z \approx 3.6$. We observed similar behavior even in $4+1 \mathrm{D}$ which is predicted by some of the macroscopic theories to be the upper critical dimension. In this case $\beta_{\text {eff }}(L) \approx 0.25$ for $L=32$ and $\zeta_{\text {eff }} \approx 0.94$ for $L=8-16$.

Another way of studying the kinetic roughening is the calculation of the height-height correlation function $G(\mathbf{r}, t)=\left\langle[h(\mathbf{x}+\mathbf{r}, t)-h(\mathbf{x}, t)]^{2}\right\rangle$. Recently, it has been found 10 , [6] that the WV model in $1+1$ and $2+1 \mathrm{D}$ exhibits anomalous scaling behavior and the exponents calculated from the surface width and from the correlation function are different. We observed this anomaly related to the increase of the average step height also in $3+1 \mathrm{D}$ and $4+1 \mathrm{D}$. The exponents $\zeta_{\text {eff }}^{\mathrm{c}}$ obtained from the correlation function at later times (when $\beta_{\text {eff }}$ is large) are: $\zeta_{\text {eff }}^{\mathrm{c}}=0.81 \pm 0.03$ in $3+1 \mathrm{D}$ and $\zeta_{\mathrm{eff}}^{\mathrm{c}}=0.7 \pm 0.1$ in $4+1 \mathrm{D}$. The sudden increase in the value of $\beta_{\text {eff }}$ described above is reflected in a faster power-law increase of the average step height $G(1, t) \propto t^{\lambda}$ with $\lambda \approx 0.08$. However, unlike in $1+1$ and $2+1 \mathrm{D}$ [6, 10], obtained values of $\zeta, \zeta^{\mathrm{c}}$ and $\lambda$ are inconsistent with the relation suggested in Ref. [10]. In other words, this anomalous behavior caused by the increase of the average step height does not explain the large values of the effective exponents which are instead caused by a mounding instability described below which leads to breakdown of conventional scaling and an evolution of a macroscopically corrugated surface.

Because of the unexpected results of our simulations, we have carefully checked possible sources of errors. The same behavior in $3+1 \mathrm{D}$ was observed using different random number generators, and even using a different code [11]. We also tried to obtain more information on the evolution of the system and studied the statistics of jumps of freshly deposited particles in different directions (jumps up and down are the most important for the developing roughness). We have found that the probability of jumps in different lateral directions is well isotropic. The number of jumps up monotonously increased up to the region of the saturated roughness where it saturated. The only quantity which seems to be related to the increase in $\beta_{\mathrm{eff}}$ is the number of jumps down, which at first rapidly increases and then, for several tens of monolayers, has a maximum and after that slowly decreases.

An important information has been provided by the study of the surface morphology 
using 2+1-dimensional sections of the 3+1-dimensional $L=50$ lattice. We observed a development of mound structures. Since the time $2^{10}$ small mounds are seen in different places which later increase in size and merge so that finally there is only one large mound in the whole sample (a typical example is shown in Fig. 3). The development of such mounds suggests the existence of an instability which we studied by calculations of a slope-dependent currents for tilted surfaces $(h \rightarrow h+\mathbf{x} \cdot \mathbf{m})$ following the paper by Krug et al. [7]. Similarly to $1+1$ and $2+1 \mathrm{D}$ (see $[7]$ ), we found a current in the downhill direction, of the order of $10^{-4}$ for the slopes $m=2$ and $m=1$. However, for small slopes $(m=0.5$ and $m=0.25)$ we observed in contrast to the lower dimensions an uphill current, which causes the instability observed (detailed results will be published elsewhere). The situation is similar to growth with the Schwoebel barrier to hopping down step edges where for small slopes a destabilizing uphill current which increases with the slope is observed whereas it monotonously decreases (but still remains uphill) for large slopes [12]. However, in our case the current changes its sign and becomes downhill for large slopes. In fact, we observed that the current is very close to zero for simulations on a surface with the average slope of $m=0.75$. Visual inspection of mounds similar to the one shown in Fig. 3 reveals that their angle of inclination is very close to the value 0.75 , in agreement with the results of the work of Krug et al. [7] according to which a slope with the zero diffusion current is selected. We do not understand, however, what drives the uphill current and why such behavior is not observed in $1+1$ and $2+1$ dimensions and these questions remain to be answered.

To study the problem further, we also performed simulations of two modifications of the WV model. At first we studied the modified WV model in which the deposition site is chosen not only from nearest-neighbor sites but from all the sites in a region of a linear dimension $R_{i}$ around the site of incidence.

We used the algorithm employed earlier in the simulation of the FD model for the relaxation of incoming particles [13. In this case, a site on the surface is selected randomly, and a site with the maximum coordination within a cube with a side of $2 R_{i}+1$ lattice constants centered upon the original site is chosen as the site of final deposition (notice that $R_{i}=1$ is different from the original WV model since also next-nearest neighbors are considered). We have observed that the roughness saturates much earlier for a larger $R_{i}$ and the value of the saturated roughness progressively decreases with increasing $R_{i}$. This makes it difficult to measure the exponent $\beta_{\text {eff }}$ for large $R_{i}$ 's; larger and larger sizes $L$ are 
needed (Fig. 4). However, within our error bars, we have obtained the same large $\beta_{\text {eff }} \approx 0.3$ for $R_{i}=1,2,3,5$. One could see that the region with an initial small value of $\beta_{\text {eff }}$ disappears with increasing $R_{i}$. The exponent $\zeta_{\text {eff }}(L=40-60)$ decreases with increasing $R_{i}$ from $\zeta_{\mathrm{eff}} \approx 1.18$ for $R_{i}=1$ to $\zeta_{\mathrm{eff}} \approx 0.69$ for $R_{i}=5$. Similarly, the exponent $\zeta_{\mathrm{eff}}^{\mathrm{c}}$ obtained from the height-height correlation function decreases with increasing $R_{i}$ from $\zeta_{\text {eff }}^{\mathrm{c}} \approx 0.82$ for $R_{i}=1$ to $\zeta_{\mathrm{eff}}^{\mathrm{c}} \approx 0.5$ for $R_{i}=3$. The decrease of the surface width is on the microscopic level reflected by the increase in the number of jumps down (and the disappearance of the small initial $\beta_{\text {eff }}$ by the disappearance of the maximum in the number of jumps down). We also studied another modification of the WV model in which jumps up are forbidden (model II of Ref. [8]) and observed a similar behavior with an increase to a large $\beta_{\text {eff }} \approx 0.25$ after $\approx 10^{2}$ layers were deposited. In both considered modifications, the roughness is lower than in the original WV model, but the mounding instability is still present.

In conclusion, we have carried out extensive simulations of a growth model proposed by Wolf and Villain with local post-deposition relaxation in $3+1$ and $4+1 \mathrm{D}$. We have found effective exponents much larger than those expected based on results for $1+1 \mathrm{D}$ and $2+1 \mathrm{D}$. Anomalous scaling due to the power-law increase of the average step height observed in $1+1 \mathrm{D}$ and $2+1 \mathrm{D}$ (manifested in different values of the roughness exponent $\zeta_{\text {eff }}$ obtained from the surface width evolution and $\zeta_{\text {eff }}^{\mathrm{c}}$ from the height-height correlation function) is also present in the higher dimensions. However, a dominant role is played by a growth instability resulting in a macroscopically modulated surface profile with large mounds developing on the surface. Calculations of diffusion currents for tilted surfaces show a destabilizing uphill current at small slopes microscopic origin of which remains unclear. This current changes its sign (becomes downhill) as the slope increases and our results suggest that the angle of inclination of the mounds observed on the surface corresponds to the slope for which the current is zero. An increase in the radius of incorporation of a freshly arrived particle leads to a decrease of the roughness but does not suppress the instability. More work is needed to determine why the mounding instability is observed here but not in the lower dimensions.

Work performed at Imperial College is supported by Imperial College and the Research Development Corporation of Japan. P. Š. would like to thank Dr. M.R. Wilby for valuable discussions and suggestions on the model and simulation technique, and for communicating his results prior to publication. M. K. wishes to thank Prof. A.C. Levi for valuable discussions. 


\section{References}

[1] For review see: Krug J. and Spohn H., in Solids Far from Equilibrium: Growth, Morphology and Defects, Ed. C. GodRÈche, (Cambridge University Press, Cambridge) 1990, p. 479; Kotrla M., Czech. J. Phys. B, 42 (1992) 449; Meakin P., Phys. Rep., 235 (1993) 189.

[2] Villain J., J. Phys. I, 1 (1991) 19.

[3] Wolf D.E. and Villain J., Europhys. Lett., 13 (1990) 389. Later, a similar model was proposed by Das Sarma S. and Tamborenea P., Phys. Rev. Lett., 66 (1991) 325.

[4] Lai Z.-W. and Das Sarma S., Phys. Rev. Lett., 66 (1991) 2348.

[5] Tang L.-H. and Nattermann T., Phys. Rev. Lett., 66 (1991) 2899.

[6] Šmilauer P. and Kotrla M., Phys. Rev. B, 49 (1994) 5769.

[7] Krug J., Plischke M. and Siegert M., Phys. Rev. Lett., 70 (1993) 3271.

[8] Kotrla M., Levi A.C. and Šmilauer P., Europhys. Lett., 20 (1992) 25.

[9] Wilby M.R., Vvedensky D.D. and Zangwill A., Phys. Rev. B, 46 (1992) 12896; (errata) Phys. Rev. B, 47 (1993) 16068.

[10] Schroeder M., Siegert M., Wolf D.E., Shore J.D. and Plischke M., Europhys. Lett., 24 (1993) 563.

[11] Wilby M.R., unpublished.

[12] Johnson M.D., Orme C., Hunt A.W., Graff D., Sudijono J., Sander L.M. and OrR B.G., Phys. Rev. Lett., 72 (1994) 116.

[13] Clarke S., Wilby M.R. and Vvedensky D.D., Surf. Sci., 255 (1991) 91. 


\section{Figure captions}

Fig.1 - Surface width $w$ vs. time $t$ for the WV model (relaxation to nearest neighbors only) in $3+1$ D. Solid squares $L=64$, stars $L=45$, solid triangles $L=32$, open squares $L=16$, open circles $L=8$, open triangles $L=4$. Inset: saturated surface width $w_{\infty} v s$. system size for the same model.

Fig.2 - Evolution of the effective exponents $\beta_{\text {eff }}$ for the WV model in $3+1 \mathrm{D}$ for different system sizes. Solid squares $L=64$, stars $L=45$, solid triangles $L=32$.

Fig.3 - Example of the surface morphology (a $2+1$ D section of a $3+1$ D lattice) obtained for a lattice of the linear dimension $L=50$ after $2^{16}$ monolayers were deposited.

Fig.4 - Comparison of the time evolution of the surface width in the modified WV model with the different incorporation radii $R_{i}=1,2,3,5$ in $3+1 \mathrm{D}$. 